\title{
Anopheles stephensi Mosquitoes as Vectors of Plasmodium vivax and P. falciparum, Horn of Africa, 2019
}

Fitsum G. Tadesse, ${ }^{1}$ Temesgen Ashine, ${ }^{1}$ Hiwot Teka, Endashaw Esayas, Louisa A. Messenger, Wakweya Chali, Lisette Meerstein-Kessel, Thomas Walker, Sinknesh Wolde Behaksra, Kjerstin Lanke, Roel Heutink, Claire L. Jeffries, Daniel Abebe Mekonnen, Elifaged Hailemeskel, Surafel K. Tebeje, Temesgen Tafesse, Abrham Gashaw, Tizita Tsegaye, Tadele Emiru, Kigozi Simon, Eyuel Asemahegn Bogale, Gedeon Yohannes, Soriya Kedir, Girma Shumie, Senya Asfer Sabir, Peter Mumba, Dereje Dengela, Jan H. Kolaczinski, Anne Wilson, Thomas S. Churcher, Sheleme Chibsa, Matthew Murphy, Meshesha Balkew, Seth Irish, Chris Drakeley, Endalamaw Gadisa, Teun Bousema

Anopheles stephensi mosquitoes, efficient vectors in parts of Asia and Africa, were found in $75.3 \%$ of water sources surveyed and contributed to $80.9 \%$ of wild-caught Anopheles mosquitoes in Awash Sebat Kilo, Ethiopia. High susceptibility of these mosquitoes to Plasmodium falciparum and vivax infection presents a challenge for malaria control in the Horn of Africa.

Talaria control programs in Africa traditionally 1 focus on rural settings, although transmission is also a health concern in some urban settings (1).

Author affiliations: Armauer Hansen Research Institute, Addis Ababa, Ethiopia (F.G. Tadesse, T. Ashine, H. Teka, E. Esayas, W. Chali, S.W. Behaksra, D.A. Mekonnen, E. Hailemeskel, S.K. Tebeje, T. Tafesse, A. Gashaw, T. Tsegaye, T. Emiru, E.A. Bogale, G. Shumie, S.A. Sabir, E. Gadisa); Radboud University Medical Center, Nijmegen, the Netherlands (F.G. Tadesse, L. Meerstein-Kessel, K. Lanke, R. Heutink, E. Hailemeskel, S.K. Tebeje, T. Bousema); Addis Ababa University, Addis Ababa (F.G. Tadesse, D.A. Mekonnen, E. Hailemeskel); United States Agency for International Development, Addis Ababa (H. Teka, S. Chibsa, M. Murphy); London School of Hygiene and Tropical Medicine, London, UK (L.A. Messenger, T. Walker, C.L. Jeffries, K. Simon, C. Drakeley, T. Bousema); President's Malaria Initiative VectorLink Ethiopia Project, Addis Ababa (G. Yohannes, P. Mumba, M. Balkew); Oromia Regional Health Bureau, Adama, Ethiopia (S. Kedir); President's Malaria Initiative VectorLink Project, Rockville, Maryland, USA (D. Dengela); World Health Organization, Geneva, Switzerland (J.H. Kolaczinski); Liverpool School of Tropical Medicine, Liverpool, UK; (A. Wilson); Imperial College London, London (T.S. Churcher); United States President's Malaria Initiative, Atlanta, Georgia, USA (S. Chibsa, M. Murphy, S. Irish); Centers for Disease Control and Prevention, Atlanta (S. Irish)
Anopheles stephensi mosquitoes breed predominantly in urban settings, prefer water storage containers (2), and are found throughout the Horn of Africa (3). To determine susceptibility of An. stephensi mosquito vectors to infection with local Plasmodium strains, we measured their abundance in an urban area of Ethiopia and characterized their aquatic habitats, biting and resting behavior, and competence to transmit local $P$. vivax and $P$. falciparum.

Study protocol was approved by the Institutional Ethical Review Board of the Aklilu Lemma Institute of Pathobiology of Addis Ababa University (ALIPB IRB/025/2011/2019), the Oromia Regional Health Bureau (BEFO/MBTFH/1331), and AHRI/ALERT Ethics Review Committee (AF-10-015.1, PO07/19). All participants or parents/legal guardians for participants $<18$ years of age provided written informed consent. Persons who volunteered for human landing collection also provided written informed consent, were monitored for 3 weeks after collections, and if symptomatic and positive received treatment for Plasmodium according to the treatment guidelines of the country.

\section{The Study}

This study was conducted in Awash Sebat Kilo, Ethiopia, an area of perennial malaria transmission, during April-September 2019. We examined aquatic habitats for immature-stage Anopheles mosquitoes by standard dipping $(10 \times /$ site $)$ for 5 consecutive days (4). We assessed mosquito resting, feeding, and host-seeking behavior by 5 methods: CDC miniature light traps model 512 (John W. Hock Company,

${ }^{1}$ These authors contributed equally to this article. 
https:/ /www.johnwhock.com), human landing collection, pyrethrum spray sheet collection, aspiration from animal shelters, and cattle-baited traps (5). We identified adult mosquitoes by using standard keys and confirmed identification by targeted sequencing of nuclear internal transcribed spacer 2 (ITS2) and mitochondrial cytochrome oxidase subunit 1 gene (COI) (6). To generate clade topologies, we compared $A n$. stephensi mosquito DNA sequences with those in publicly available libraries (7). We determined mosquito blood meal sources by using multiplex PCR targeting cytochrome $b(8)$ and infection status by using 185 rRNA nested PCR (9).

Adult An. stephensi mosquitoes reared from immature mosquitoes from local water sources and a colony of $A n$. arabiensis mosquitoes ( $\approx 120$ each) were fed in the dark for 30 min on membrane feeders containing fresh blood from Adama malaria clinic patients with microscopy-confirmed mono- and mixedspecies infections with $P$. vivax and $P$. falciparum (10). Unfed and partially fed mosquitoes were removed; fully engorged mosquitoes were maintained on sugar solution. At 7 or 12 days after feeding, mosquitoes were dissected, their midguts were examined for oocysts, and their salivary glands were examined for sporozoites. To compare infection status between $A n$. arabiensis and $A n$. stephensi mosquitoes, we performed logistic regression. We used individual mosquito data and a fixed effect for each patient to account for correlations between mosquito observations from the same donor. Bland-Altmann plots were generated for differences in infectivity between mosquito sources by using the Pitman test of difference in variance. For analyses, we used STATA version 13 (StataCorp., https://www.stata.com/company) and GraphPad Prism 5.3 (GraphPad Software Inc., https://www. graphpad.com). Raw data have been deposited in the DRYAD data depository (https://datadryad.org/ stash/dataset/doi:10.5061/dryad.gf1vhhmnt).

An. stephensi larvae were detected in $75.3 \%$ $(64 / 85)$ of the 85 artificial water sources surveyed (Table 1). A total of 49,393 immature Anopheles larvae and pupae were collected during 20 weekly collections in April-September 2019, of which 45,316 $(91.7 \%)$ emerged as adult mosquitoes in the laboratory. Morphologic identification of adults confirmed that all were An. stephensi. During monthly rounds of entomologic surveillance in August and September (6 days each), we collected 89 adult female Anopheles mosquitoes (72 [80.9\%] An. stephensi, 16 An. gambiae, and 1 An. pharoensis). We detected $P$. vivax in $2.8 \%$ $(2 / 72)$ and $P$. falciparum in $1.4 \%(1 / 72)$ of wild-caught An. stephensi mosquitoes. Blood meal source was identified for $35.0 \%$ (28/80) blood-fed wild-caught An. stephensi mosquitoes; exclusive human blood meal was identified for $17.2 \%(5 / 29)$. The remainder fed (multiple blood meals) either on humans and animals (n $=9)$ or animals only $(n=14)$ such as goats $(n=21)$, cows $(n=4)$, and dogs $(n=5)$. Successful sequencing of ITS2 for 76 and COI for 45 Anopheles mosquitoes confirmed that all were An. stephensi. According to ITS2 sequences, An. stephensi mosquitoes from Ethiopia formed a well-supported monophyletic clade with isolates from the Arabian Peninsula and Southeast

\begin{tabular}{|c|c|c|c|}
\hline Characteristic & Habitats, no. & $\begin{array}{c}\text { Mosquito larvae, no. larvae } \\
\text { detected/no. habitats sampled (\%) }\end{array}$ & $\begin{array}{c}\text { Mosquito pupae, no. pupae } \\
\text { detected/no. habitats sampled (\%) }\end{array}$ \\
\hline \multicolumn{4}{|c|}{ Localities (kebeles) within the town of Awash Sebat Kilo } \\
\hline Sebat Kilo & 60 & $44 / 60(73.3)$ & $19 / 44(43.2)$ \\
\hline Lemlefan & 17 & $12 / 17(70.6)$ & $0 / 12(0)$ \\
\hline Alalamo & 8 & $8 / 8(100.0)$ & $5 / 8(62.5)$ \\
\hline \multicolumn{4}{|l|}{ Artificial containers } \\
\hline Permanent & 48 & $41 / 48(85.4)$ & $17 / 41(41.5)$ \\
\hline Temporary & 37 & $23 / 37$ (62.2) & $7 / 23(30.4)$ \\
\hline \multicolumn{4}{|l|}{ Shade status } \\
\hline Fully & 22 & 14/22 (63.6) & $6 / 14(42.9)$ \\
\hline Partial & 24 & $22 / 24(99.7)$ & $7 / 22(31.8)$ \\
\hline None & 39 & $28 / 39(71.8)$ & $11 / 28(39.3)$ \\
\hline \multicolumn{4}{|l|}{ Use status } \\
\hline In use & 71 & $54 / 71(76.1)$ & 20/54 (37.0) \\
\hline Not in use & 14 & $10 / 14(71.4)$ & $4 / 10(40.0)$ \\
\hline \multicolumn{4}{|l|}{ Container material } \\
\hline Fiber jar/tire & 23 & $10 / 23(43.5)$ & $4 / 10(40.0)$ \\
\hline Metal/steel tanks/drum/barrel & 17 & 16/17 (94.1) & $5 / 16(31.3)$ \\
\hline Cement/ceramic & 45 & $38 / 4(84.4)$ & $15 / 38(39.5)$ \\
\hline \multicolumn{4}{|l|}{ Water turbidity } \\
\hline Clean & 56 & 45/56 (80.4) & $17 / 45$ (37.8) \\
\hline Turbid & 28 & 19/28 (67.9) & $7 / 19(36.8)$ \\
\hline
\end{tabular}


Table 2. Characteristics of blood meals and mosquito feeding outcomes in study of Anopheles stephensi mosquitoes as vectors of Plasmodium vivax and falciparum, Horn of Africa, 2019*

\begin{tabular}{|c|c|c|c|}
\hline \multirow[b]{2}{*}{ Characteristic } & \multicolumn{3}{|c|}{ Plasmodium species } \\
\hline & P. vivax, $\mathrm{n}=36$ & P. falciparum, $\mathrm{n}=7$ & Mixed, $n=4$ \\
\hline Parasites/ $\mu \mathrm{L}$, median (IQR) & $7,783(3,603-13,440)$ & $2,431(867-8,756)$ & $4,516(1,589-10,563)$ \\
\hline Gametocyte positivity, no. positive/no. sampled (\%) & $25 / 34(73.5)$ & $1 / 7(14.3)$ & $1 / 4(25.0)$ \\
\hline Infectious feeds, no. positive/no. sampled (\%) & $26 / 36(72.2)$ & $1 / 7(14.3)$ & $2 / 4(50.0)$ \\
\hline $\begin{array}{l}\text { Infected An. stephensi mosquitoes, no. positive/no. } \\
\text { sampled (\%) }\end{array}$ & $446 / 849(52.5$ & 2.2 & $36 / 104(34.6)$ \\
\hline $\begin{array}{l}\text { Infected An arabiensis mosquitoes, no. positive/no. } \\
\text { sampled (\%) }\end{array}$ & $452 / 1,000(45.2)$ & $18 / 200(9.0)$ & $45 / 122(36.9)$ \\
\hline $\begin{array}{l}\text { Oocysts in infected } A n \text {. arabiensis mosquito midgut, mean } \\
\text { (range) }\end{array}$ & $22.8(1-115)$ & NA & $3.1(1-22)$ \\
\hline $\begin{array}{l}\text { Oocysts in infected An. stephensi mosquito midgut, mean } \\
\text { (range) }\end{array}$ & $24.1(1-105)$ & NA & $2.8(1-13)$ \\
\hline
\end{tabular}

Asia (Appendix, https://wwwnc.cdc.gov/EID/ article/27/2/20-0019-App1.pdf). The COI tree was more resolutive, suggesting that An. stephensi mosquitoes from Ethiopia were most closely related to mosquitoes from Djibouti (64\%) and Pakistan (54\%).

We conducted 47 paired-membrane feeding experiments by using blood from patients with microscopy-confirmed $P$. vivax or $P$. falciparum infection (Table 2). The proportion of blood-fed mosquitoes was generally higher for An. arabiensis (median 80.5\%; interquartile range [IQR] 72.5-85.0) than An. stephensi mosquitoes (median 53.5\%, IQR 44.0-68.0; p<0.001; Figure 1, panel A). The proportions of the 2 mosquito species infected with $P$. vivax were strongly associated $(\rho=0.82, p<0.001$; Figure 1, panel B); a significantly higher proportion of An. stephensi (median 75.1\%, IQR 60.0-85.9) than An. arabiensis mosquitoes were infect- ed (median 58.4\%, IQR 40.0-85.6; p<0.042). Allowing for the number of dissected mosquitoes for each set of paired feeding experiments, the odds of an individual mosquito becoming infected was higher for An. stephensi mosquitoes (odds ratio [OR] 1.99, 95\% CI 1.52-2.59; $\mathrm{p}<0.001$ ) (Figure 1, panel C). The number of oocysts per infected midgut was also higher for An. stephensi (median 17, IQR 6-33) than An. arabiensis mosquitoes (median 13, IQR 4-30); $\mathrm{p}<0.001$ (Figure 2, panel A). The number of oocysts was positively associated with the proportion of infected mosquitoes for An. stephensi $(\rho=0.553, p<0.001)$ and An. arabiensis mosquitoes $(\rho=0.576, p<0.001$; Figure 2, panel B). Among paired feedings, sporozoites were detected in 52.2\% (47/90) An. arabiensis and $75.0 \%(84 / 112)$ An. stephensi mosquitoes. A much higher proportion of An. stephensi $(51.8 \%, 58 / 112)$ than An. arabiensis
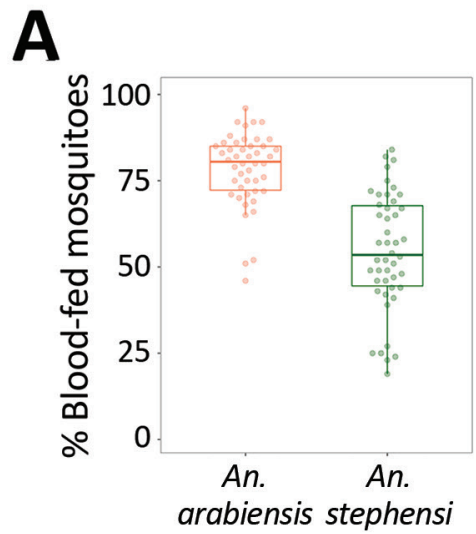

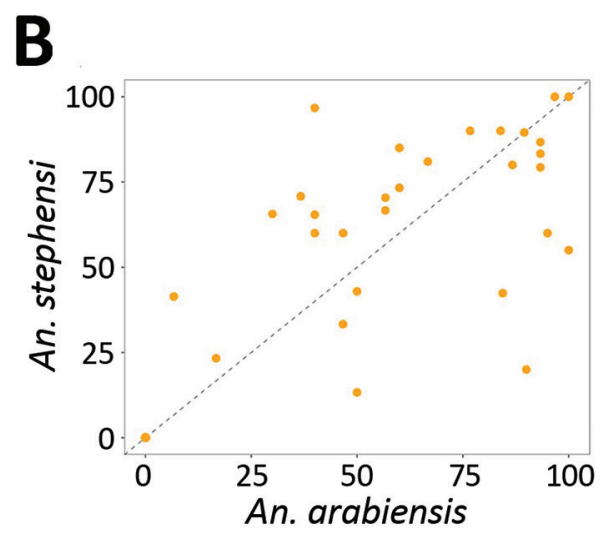

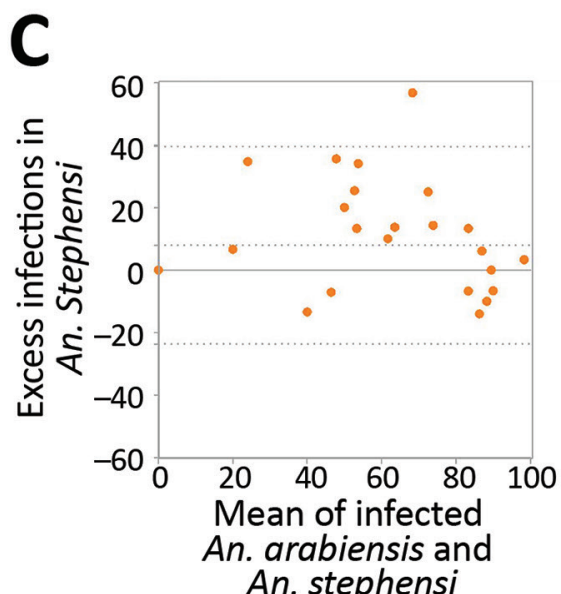

An. stephensi

Figure 1. Comparison of feeding efficiency and infection rates for Anopheles stephensi and An. arabiensis mosquitoes in paired feeding experiments in study of An. stephensi mosquitoes as vectors of Plasmodium vivax and falciparum, Horn of Africa, 2019. A) Percentage of fully fed An. arabiensis mosquitoes (red) and An. stephensi mosquitoes (green). Box plots indicate median (midline), 25th (lower line), and 75th (upper line) percentiles of proportion of blood-fed mosquitoes. Whiskers indicate lower and upper $25 \%$ scores. Vertical lines indicate minimum and maximum values. B) Percentage of infected mosquitoes. C) Bland-Altman plot (difference plots) for mosquito infection rates in different mosquito species. Symbols indicate differences in infection rates in An. stephensi versus An. arabiensis (y-axis) mosquitoes in relation to mean infection rates in these 2 species (x-axis). Positive values $(57.1 \% ; 16 / 28)$ indicate a higher infection rate in An. stephensi mosquitoes; dotted lines indicate the $95 \%$ limits of agreement. There was no evidence that the correlation coefficient between the paired differences and means differed significantly from 0 (Pitman test of difference in variance, $r=0.026, p=0.864$ ). 
A

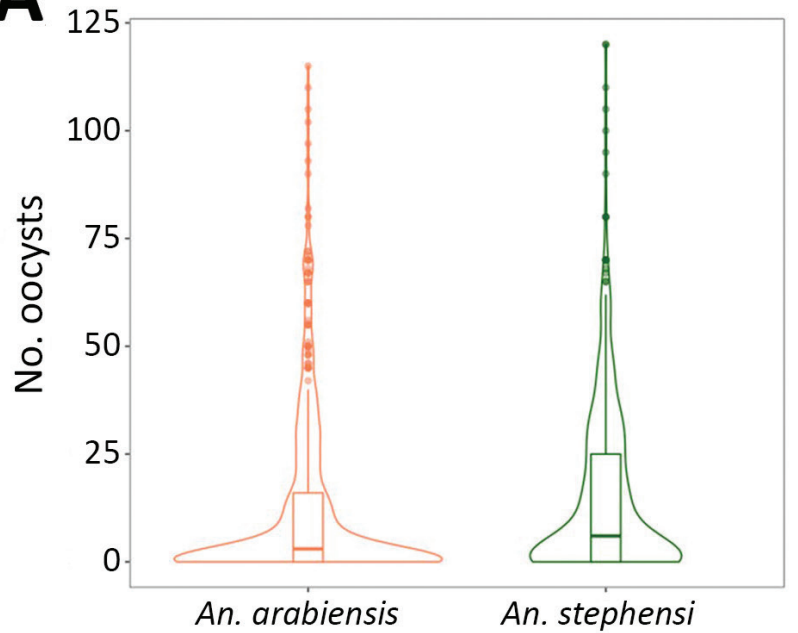

B

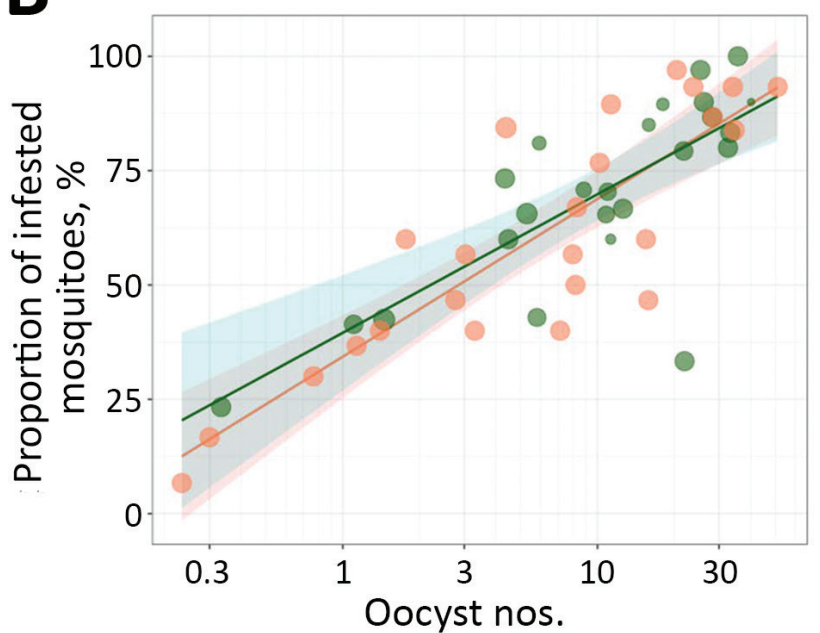

Figure 2. Comparison of relative oocyst numbers and infection rate for Anopheles stephensi and An. arabiensis mosquitoes in paired feeding experiments in study of An. stephensi mosquitoes as vectors of Plasmodium vivax and falciparum, Horn of Africa, 2019. Number of oocysts per infected midgut for individual mosquitoes of each of the 2 species. A) Violin plot showing estimated kernel density. Horizontal lines indicate median; box indicates interquartile range; and spikes indicate upper and lower adjacent values. The proportion of midguts with detectable oocysts (y-axis) is indicated in association with $\log _{10}$ transformed oocyst numbers (x-axis) for An. stephensi (green dots) and An. arabiensis (orange dots) mosquitoes. B) Data for 24 feeding experiments in which 723 An. arabiensis and 643 An. stephensi mosquitoes were dissected. Shaded area indicates $95 \% \mathrm{Cl}$ around estimates for An. stephensi (green) and An. arabiensis (orange) mosquitoes.

mosquitoes $(31.1 \%, 28 / 90)$ had high sporozoite load $(+3$ and +4$) ; p=0.011$. After accounting for the number of examined salivary glands, the odds of detecting high sporozoite intensity were substantially higher for An. stephensi than An. arabiensis mosquitoes (OR $4.6,95 \%$ CI 2.2-9.9; $\mathrm{p}<0.001)$.

\section{Conclusions}

An. stephensi mosquitoes have spread from Asia throughout the Horn of Africa, detected in Djibouti in 2012 (11), Ethiopia in 2016 (12), and Sudan in 2019 (3). The widescale presence of An. stephensi mosquitoes in developmental stages in artificial water bodies demonstrates that these mosquitoes are firmly established in an urban setting in Ethiopia, located on the main transportation corridor from Djibouti to Addis Ababa. Detection of 4 haplotypes suggests independent arrival of different populations or heterogeneity arising after importation of the mosquito species. Our mosquito feeding experiments predominantly included highly infective patients with clinical P. vivax infection $(10,13)$. Although feeding rates for the membrane-adapted colony of An. arabiensis mosquitoes were high, mosquito infection rates were significantly higher for An. stephensi mosquitoes. Our detection of salivary gland sporozoites establishes that sporogonic development of local P. vivax can be completed by An. stephensi mosquitoes. We recruited fewer patients with clinical P. falciparum infection, who were less likely than $P$. vivax patients to infect mosquitoes (10). Despite a modest number of observations, our findings demonstrate that local $P$. falciparum isolates are also capable of infecting An. stephensi mosquitoes and are further supported by detection of P. falciparum- and P. vivax-infected wild-caught adult mosquitoes.

Spread of An. stephensi mosquitoes poses risk for increased $P$. falciparum and $P$. vivax receptivity and local transmission in urban Africa. Given mosquito preference for human-made containers (14), our findings support integrated vector management recommended by the World Health Organization under the Global Vector Control Response (15). Management may include integrated surveillance and control of other vectors such as Aedes aegypti mosquitoes for larval source management.

\section{Acknowledgments}

We acknowledge the study participants for their willingness to donate blood and allow assessment of mosquito exposure. We thank microscopists Tewabech Lema and Tsehay Orlando for their support. Appreciation also goes to the regional and district health officers for their collaboration and the PMI VectorLink Ethiopia Project for training the field team. Thanks also go to Gunawardena Dissanayake for insightful discussions. The 
drivers from Armauer Hansen Research Institute helped make the study successful.

The study was supported by the Bill and Melinda Gates Foundation grant (INDIE OPP1173572) to F.G.T., T.B., and C.D. The Armauer Hansen Research Institute supported W.C., T.A., E.H., and E.G. through its core funding from the Swedish International Development Cooperation Agency and the Norwegian Agency for Development Cooperation. The PMI VectorLink Ethiopia Project provided financial support for adult mosquito collection. C.L.J. and T.W. were supported by Wellcome Trust/Royal Society fellowships awarded to T.W. (101285/Z/13/Z).

H.T., P.M., S.C., M.M., M.B., S.I., C.D., E.G., T.B., and F.G.T. conceived the study; P.M., D.D., S.C., M.M., M.B., S.I., J.H.K., A.W., E.G., and F.G.T. participated in guiding the field activities; T.A. and E.E. collected mosquitoes in developmental stages; T.A., E.E., W.C., S.W.B., D.A.M., E.H., S.K.T., T.T., A.G., T.T., T.E., G.Y., S.K., G.S., and S.A.S. reared adult mosquitoes, collected blood samples, ran feeding experiments, and dissected mosquitoes; T.A., H.T., E.E., L.A.M., W.C., T.W., S.W.B., K.L., R.H., C.L.J., D.A.M., E.H., S.K.T., T.T., A.G., T.T., T.E., and F.G.T. conducted laboratory work; T.A., L.A.M., L.M.K., K.S., T.S.C., S.I., C.D., E.G., T.B., and F.G.T. analyzed data; T.A., H.T., E.E., L.A.M., T.W., C.L.J., C.D., E.G., T.B., and F.G.T. drafted the manuscript; and J.H.K., A.W., T.S.C., S.I., C.D., E.G., T.B., F.G.T. critically commented on the manuscript. All authors read and approved the final manuscript.

\section{About the Author}

Dr. Tadesse is a molecular biologist leading the malaria research team of the Armauer Hansen Research Institute at the Federal Ministry of Health of Ethiopia and teaches at the Institute of Biotechnology at Addis Ababa University. His research interests include the biology and epidemiology of gametocytes of $P$. vivax and $P$. falciparum.

\section{References}

1. Wilson ML, Krogstad DJ, Arinaitwe E, Arevalo-Herrera M, Chery L, Ferreira MU, et al. Urban malaria: understanding its epidemiology, ecology, and transmission across seven diverse ICEMR network sites. Am J Trop Med Hyg. 2015;93(Suppl):110-23. https:// doi.org/10.4269/ ajtmh.14-0834

2. Surendran SN, Sivabalakrishnan K, Sivasingham A, Jayadas TTP, Karvannan K, Santhirasegaram S, et al. Anthropogenic factors driving recent range expansion of the malaria vector Anopheles stephensi. Front Public Health. 2019;7:53. https://doi.org/10.3389/fpubh.2019.00053

3. World Health Organization. Vector alert: Anopheles stephensi invasion and spread [cited 2020 Sep 3]. https:/ / www.who. int/publications/i/item/vector-alert-anopheles-stephensiinvasion-and-spread

4. World Health Organization. Larval source management: a supplementary measure for malaria vector control: an operational manual [cited 2020 Dec 21]. https:/ / apps.who.int/ iris/bitstream/handle/10665/85379/9789241505604_eng.pdf

5. St Laurent B, Oy K, Miller B, Gasteiger EB, Lee E, Sovannaroth $S$, et al. Cow-baited tents are highly effective in sampling diverse Anopheles malaria vectors in Cambodia. Malar J. 2016;15:440. https://doi.org/10.1186/ s12936-016-1488-y

6. Folmer O, Black M, Hoeh W, Lutz R, Vrijenhoek R. DNA primers for amplification of mitochondrial cytochrome $\mathrm{C}$ oxidase subunit I from diverse metazoan invertebrates. Mol Mar Biol Biotechnol. 1994;3:294-9.

7. Kumar S, Stecher G, Li M, Knyaz C, Tamura K. MEGA X: Molecular Evolutionary Genetics Analysis across computing platforms. Mol Biol Evol. 2018;35:1547-9. https:/ / doi.org/ 10.1093/molbev/msy096

8. Kent RJ, Norris DE. Identification of mammalian blood meals in mosquitoes by a multiplexed polymerase chain reaction targeting cytochrome B. Am J Trop Med Hyg. 2005;73:33642. https://doi.org/10.4269/ajtmh.2005.73.336

9. Snounou G, Viriyakosol S, Zhu XP, Jarra W, Pinheiro L, do Rosario VE, et al. High sensitivity of detection of human malaria parasites by the use of nested polymerase chain reaction. Mol Biochem Parasitol. 1993;61:315-20. https://doi.org/10.1016/0166-6851(93)90077-B

10. Tadesse FG, Slater HC, Chali W, Teelen K, Lanke K, Belachew $\mathrm{M}$, et al. The relative contribution of symptomatic and asymptomatic Plasmodium vivax and Plasmodium falciparum infections to the infectious reservoir in a lowendemic setting in Ethiopia. Clin Infect Dis. 2018;66:1883-91. https://doi.org/10.1093/cid/cix1123

11. Faulde MK, Rueda LM, Khaireh BA. First record of the Asian malaria vector Anopheles stephensi and its possible role in the resurgence of malaria in Djibouti, Horn of Africa. Acta Trop. 2014;139:39-43. https://doi.org/10.1016/ j.actatropica.2014.06.016

12. Carter TE, Yared S, Gebresilassie A, Bonnell V, Damodaran L, Lopez K, et al. First detection of Anopheles stephensi Liston, 1901 (Diptera: culicidae) in Ethiopia using molecular and morphological approaches. Acta Trop. 2018;188:180-6. https://doi.org/10.1016/j.actatropica.2018.09.001

13. Kiattibutr K, Roobsoong W, Sriwichai P, Saeseu T, Rachaphaew N, Suansomjit C, et al. Infectivity of symptomatic and asymptomatic Plasmodium vivax infections to a Southeast Asian vector, Anopheles dirus. Int J Parasitol. 2017;47:163-70. https:// doi.org/10.1016/j.ijpara.2016.10.006

14. Surendran SN, Sivabalakrishnan K, Gajapathy K, Arthiyan S, Jayadas TTP, Karvannan K, et al. Genotype and biotype of invasive Anopheles stephensi in Mannar Island of Sri Lanka. Parasit Vectors. 2018;11:3. https:// doi.org/10.1186/s13071-017-2601-y

15. World Health Organization. Global vector control response 2017-2030 [cited 2020 Jun 7]. https:/ / www.who.int/ vector-control/publications/global-control-response/en/

Address for correspondence: Fitsum G. Tadesse, Malaria and NTD directorate, Armauer Hansen Research Institute, Federal Ministry of Health, PO Box 1005, Addis Ababa, Ethiopia; email: fitsum.tadesse@radboudumc.nl 